\title{
ESTILOS DE VIDA EM TRABALHADORES OFFSHORE DE UMA PLATAFORMA DA ÁFRICA SUBSAARIANA
}

\author{
Eduardo Miguel Coelho \\ Hospital São Pedro de Vila Real (Centro Hospitalar de Trás-os-Montes e Alto Douro) \\ Serviço de Urgência, Portugal \\ Odete Amaral \\ Escola Superior de Saúde de Viseu, Instituto Politécnico de Viseu. CI\&DETS, Portugal \\ mopamaral@gmail.com \\ Carlos Pereira \\ Escola Superior de Saúde de Viseu, Instituto Politécnico de Viseu. CI\&DETS, Portugal \\ Nélio Veiga \\ Health Sciences Institute - Universidade Católica Portuguesa Viseu, Portugal. \\ Centre for Interdisciplinary Health Research (CIIS) - Universidade Católica Portuguesa, Portugal. \\ José Costa \\ Escola Superior de Saúde de Viseu, Instituto Politécnico de Viseu. CI\&DETS, Portugal \\ Paula Nelas \\ Escola Superior de Saúde de Viseu, Instituto Politécnico de Viseu. CI\&DETS, Portugal \\ Cláudia Chaves \\ Escola Superior de Saúde de Viseu, Instituto Politécnico de Viseu. CI\&DETS, Portugal \\ Emília Coutinho \\ Escola Superior de Saúde de Viseu, Instituto Politécnico de Viseu. CI\&DETS, Portugal
}

Fecha de Recepción: 4 Febrero 2018 Fecha de Admisión: 10 Abril 2018

\begin{abstract}
RESUMO
Introdução: 0 trabalhador offshore está confinado a um espaço limitado durante um período de tempo. Investigar como as características do ambiente de trabalho afetam a saúde dos trabalhadores constitui uma preocupação. 0 objectivo deste estudo foi avaliar os estilos de vida em trabalhadores offshore de uma plataforma da África Subsaariana. Participantes e Métodos: Estudo transversal com uma amostra de 99 trabalhadores em offshore, totalidade do sexo masculino, uma média de idade de $39,11 \pm 6,91$ anos; $78,5 \%$ casados e $83,8 \%$ possui entre $05^{\circ}-12^{\circ}$ ano de escolaridade. Os dados foram recolhidos através de um questionário auto-aplicado constituído por variáveis sociodemográficas, profissionais e referentes a estilos de vidas. Os dados foram analisados com recurso ao SPSS versão 24 para Windows. Resultados: Dos trabalhadores em offshore, $54,2 \%$ referiu ter 3 ou mais filhos; $54,5 \%$ ter uma mulher e $24,2 \%$ duas mulheres; $33,3 \%$ indicou possuir duas
\end{abstract}




\section{ESTILOS DE VIDA EM TRABALHADORES OFFSHORE DE UMA PLATAFORMA DA ÁFRICA SUBSAARIANA}

residências. Quanto às variáveis profissionais, em média, o número de anos de trabalho em offshore foi de $10,07 \pm 5,52$ anos e $75,8 \%$ dos trabalhadores realiza um sistema de rotação de 4 semanas. Estilos de vida, $54,5 \%$ refere que toma café diariamente, $20 \%$ que toma café depois das 20 horas, em média bebem $3,37 \pm 1,83$ cafés por dia; $74,4 \%$ refere que consome diariamente refrigerantes, 0 mais consumido a coca-cola $(67,6 \%)$; quase a totalidade dos trabalhadores consome bebidas alcoólicas em terra (83,8\%), sendo a mais consumida cerveja (68,7\%); $22,2 \%$ fuma e em média fumam $15,64 \pm 8,52$ cigarros por dia. Mais de metade da amostra possui pré-obesidade $(63,6 \%)$ e 19,2\% obesidade; $45,5 \%$ refere que às vezes pratica exercício físico e $22,2 \%$ frequentemente. Nos últimos 12 meses, $72,0 \%$ dos trabalhadores consumiu medicação para dormir; $14,0 \%$ sofre de alguma doença, sendo a mais prevalente a hipertensão (42,9\%); nos últimos 12 meses 91,0\% da amostra consultou um médico e 78,0\% consultou um médico de clínica geral. Conclusões: Encontrámos elevadas prevalências de estilos de vida pouco saudáveis, consumos, falta de exercício físico regular, excesso de peso e, quase metade dos trabalhadores sofre de hipertensão.

Palavras chave: adulto; estilo de vida; trabalhadores; categorias de trabalhadores; saúde do trabalhador

\section{ABSTRACT}

Lifestyles in offshore workers from a sub-Saharan Africa platform.

Background: The offshore worker is confined to a limited space during a certain period of time. Investigating how the characteristics of the work environment affect workers' health is a concern. The objective of this study was to assess the lifestyles of offshore workers from a sub-Saharan Africa platform. Participants and Methods: A cross-sectional study with a sample of 99 offshore workers, all of the male gender, with mean age $39.11-11.69$ years; $78.5 \%$ are married and $83.8 \%$ have scholarship between the 5th and 12th grades. Data collection was accomplished through a selfadministered questionnaire consisting of sociodemographic, professional and lifestyle variables. Data were analyzed using SPSS version 24 for Windows. Results: Of the offshore workers, $54.2 \%$ reported having 3 or more children; $54.5 \%$ have one woman and $24.2 \%$ have two women; $33.3 \%$ indicated to have two residences. Regarding the professional variables, on average, the number of years working offshore was $10.07 \pm 5.52$ years and $75.8 \%$ of the workers performed a rotation system of 4 weeks. Referring to lifestyle variables, $54.5 \%$ reported drinking coffee daily, $20 \%$ having coffee after 20 hours, on average drinking 3.37-1.83 coffees a day; $74.4 \%$ reported that they consume soft drinks daily, the most consumed soft drink was coca-cola $(67.6 \%)$; almost all workers consumed alcoholic beverages on land $(83.8 \%)$, with the most consuming mainly beer $(68.7 \%)$; $22.2 \%$ smoke and on average smoke $15.64 \pm 8.52$ cigarettes per day. More than half of the sample had pre-obesity (63.6\%) and $19.2 \%$ obesity; $45.5 \%$ reported that they sometimes exercise and $22.2 \%$ frequently exercise. In the last 12 months, $72.0 \%$ of workers consumed sleeping pills; $14.0 \%$ suffer from some kind of disease, the most prevalent being hypertension $(42.9 \%)$; in the last 12 months $91.0 \%$ of the sample consulted a physician and $78.0 \%$ consulted a general practitioner. Conclusions: We found high prevalences of unhealthy lifestyles, consumptions, lack of regular exercise, overweight and almost half of the workers suffer from hypertension.

Keywords: adults; lifestyle; workers; occupational groups; occupational health

\section{INTRODUÇÃO}

0 trabalho offshore ${ }^{1}$ pode ser caracterizado um processo/operação contínuo, sem interrupções durante 365 dias do ano, onde apenas o que é substituído e rotativo de forma ininterrupta é a mãode-obra (Leite, 2009). 0 que diferencia o trabalho offshore de outros trabalhos é o facto de 0 trabalhador offshore estar confinado a um espaço limitado/restrito, organizado por turnos, termina 0 
serviço e não pode ir para casa, para junto da família, pois continua confinado durante o período de descanso, imerso nas atividades e no ambiente de trabalho. Por 'confinamento' entende-se a limitação de espaço, um isolamento social, distanciamento da família principalmente em eventos significativos e simbólicos, ausência de privacidade enquanto embarcado e abstinência sexual forçada (Castro \& Nunes, 2008). 0 trabalho em plataforma reflete estes conspectos e 0 sistema de rotação que consiste no período de dias embarcado no mar (offshore) e o período de dias de descanso em terra (onshore). Comummente, o horário de rotação mais praticado é o de 14 dias de confinamento e 14 dias de folga em onshore. Contudo, há outras plataformas que apresentam outros sistemas de rotação, de 4 em 4 semanas ou períodos em que os marítimos ficam embarcados 28 dias, sendo os 28 dias seguintes para descanso em terra. Nesses 28 dias embarcados, cumprem regime de trabalho de 12 horas diárias, sendo 6 horas de trabalho, seis de descanso e mais seis de trabalho (0 chamado "sistema seis por seis") (Maciel de Carvalho, 2010). No final do dia de trabalho, os trabaIhadores permanecem na plataforma, num ambiente restrito para a realização das atividades de lazer e sono além de, necessariamente vivenciarem a interface casa trabalho de forma diferenciada de outros trabalhadores (Parkes, 2007).

0 trabalhador em espaços confinados apresenta um conjunto de fatores de risco que colocam em causa a segurança e a saúde do trabalhador. 0 trabalho em plataformas, desempenhado por turnos e em confinamento acarreta graves consequências para a saúde do trabalhador a nível mental, social e físico diminuindo o desempenho para a realização das tarefas e aumentando o risco de acidentes laborais (Silva, Júnior \& Ferreira, 2007; Antoniolli, Emmel, Ferreira, Paz \& Kaiser, 2015). Assim, as plataformas constituem um meio ambiente propício a determinados estilos de vida prejudiciais para a saúde pessoal e da "empresa" (Silva, Júnior \& Ferreira, 2007; Antoniolli, Emmel, Ferreira, Paz \& Kaiser, 2015). Estes trabalhadores apresentam-se mais vulneráveis para doenças físicas, psíquicas, ao stresse, o que pode influenciar a vida profissional, pessoal dos trabalhadores e os custos para a empresa.

As doenças do trabalhador embarcado estão relacionadas, sobretudo, com estilos de vida que adoptam devido às características e exigências do próprio trabalho, uma alimentação inadequada, obesidade, fumo, álcool e sedentarismo (Felipe-de-Melo, Silva, Assis \& Pinto, 2011; Oenning, Carvalho \& Lima, 2014).

Um estudo realizado em 2014 com uma amostra de 210 trabalhadores das instalações petrolí-

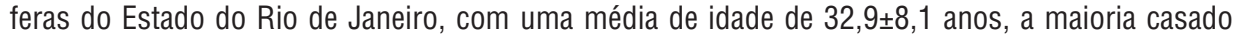
(62,9\%), 20,3\% com ensino superior, demonstrou que 15,2\% apresentavam consumo abusivo de álcool, 30,2\% fumava e 56,6\% tinham menos de 5 anos de experiência no campo offshore (Vidal, Abreu \& Portela, 2017). Um estudo caso-controlo realizado com 120 casos e 656 controles de uma empresa de petróleo na Região Norte-Nordeste do Brasil entre 2007 e 2009, revelou uma associação significativa entre 0 absenteísmo por doença e as variáveis sexo (feminino $0 R=2,54 ; p<0,0001$ ), tabagismo (fumador $O R=2,00 ; p=0,04$; ex-fumador $O R=1,70 ; p=0,02)$, sono $(0 R=2,37 ; p<0,0001$ ) (Oenning, Carvalho \& Lima, 2014).

Assim, o objectivo deste estudo foi avaliar os estilos de vida em trabalhadores offshore de uma plataforma da África Subsaariana.

\section{PARTICIPANTES E MÉTODOS}

Realizámos um estudo transversal. A população de trabalhadores da plataforma da África Subsaariana foi de 120 trabalhadores e o espaço amostral ficou constituído por 102 trabalhadores. Dos questionários recolhidos $(n=102)$ foram excluídos dois por se encontrarem incompletos e um porque era o único do sexo feminino, ficando a amostra total composta por 99 trabalhadores de uma 
plataforma da África Subsaariana. Assim, a amostra na sua totalidade era do género masculino e com uma média de idades de 39,11 $\pm 6,91$ anos (variando entre os 26 e os 59 anos). A maior percentagem dos trabalhadores apresentava menos de 35 anos (31,3\%); eram casados ou viviam em união de facto (75,8\%); 83,8\% possuía entre $05^{-0}-12^{\circ}$ ano de escolaridade e $89,9 \%$ era de nacionalidade angolana (Tabela 1). Quanto às variáveis familiares, a maioria dos trabalhadores referiu ter uma mulher $(54,5 \%)$ e $24,2 \%$ duas mulheres; mais de metade dos trabalhadores tinha três ou mais filhos (54,5\%); 46,5\% dos trabalhadores possuía uma residência e 33,3\% mencionou possuir duas residências (Cf. Tabela 1).

Os dados foram recolhidos através de um questionário autoaplicado, sendo constituído por questões para avaliação das características sociodemográficas, familiares, profissionais e de estilos de vida. Foram consideradas as seguintes variáveis relacionadas aos estilos de vida: consumo de café, tabagismo, álcool, consumo de refrigerantes, padrão de consumo de álcool em terra, a prática de exercício físico e o índice de massa corporal (IMC).

0 instrumento de colheita de dados foi submetido à Comissão de Ética da Escola Superior de Saúde de Viseu, autorização Parecer 28/2015, à Offshore Installation Manager (OIM) e foi assinado 0 consentimento informado por todos os trabalhadores da plataforma. Foram respeitados o direito à autodeterminação, intimidade, ao anonimato e à confidencialidade.

Antes da aplicação do questionário realizou-se um pré-teste a quatro trabalhadores a fim de avaliar a compreensão das questões, por forma a evitar incompreensões e equívocos, sendo que não foi necessário realizar qualquer tipo de reajustamento e reformulação de questões.

Tabela 1 - Caracterização sociodemográfica e familiar da amostra

\begin{tabular}{|c|c|c|}
\hline & $\begin{array}{c}\mathrm{n} \\
(99)\end{array}$ & $\begin{array}{c}\% \\
(100 \%) \\
\end{array}$ \\
\hline \multicolumn{3}{|l|}{ Idade } \\
\hline$<35$ anos & 31 & 31,3 \\
\hline 35-39 anos & 25 & 25,3 \\
\hline $40-44$ anos & 25 & 25,3 \\
\hline$\geq 45$ anos & 18 & 18,2 \\
\hline \multicolumn{3}{|l|}{ Estado civil } \\
\hline Solteiro / separado & 24 & 24,2 \\
\hline Casado / união de facto & 75 & 75,8 \\
\hline \multicolumn{3}{|l|}{ Habilitações Literárias } \\
\hline $1^{\circ}$ ciclo & 8 & 8,1 \\
\hline $2^{\circ}$ e $3^{\circ}$ ciclo e secundário & 83 & 83,8 \\
\hline Curso profissional / superior & 8 & 8,1 \\
\hline \multicolumn{3}{|l|}{ Nacionalidade } \\
\hline Angolana & 89 & 89,9 \\
\hline Portuguesa & 10 & 10,1 \\
\hline \multicolumn{3}{|l|}{ Número de Filhos } \\
\hline Sem filhos & 7 & 7,1 \\
\hline 1 filho & 11 & 11,1 \\
\hline 2 filhos & 27 & 27,3 \\
\hline$\geq 3$ filhos & 54 & 54,5 \\
\hline \multicolumn{3}{|l|}{ Número de Mulheres } \\
\hline Sem mulheres & 10 & 10,1 \\
\hline 1 mulher & 54 & 54,5 \\
\hline 2 mulheres & 24 & 24,2 \\
\hline$\geq 3$ mulheres & 11 & 11,1 \\
\hline \multicolumn{3}{|l|}{ Número de residências } \\
\hline Nenhuma residência & 4 & 4,0 \\
\hline 1 residência & 46 & 46,5 \\
\hline 2 residências & 33 & 33,3 \\
\hline$\geq 3$ residências & 16 & 16,2 \\
\hline
\end{tabular}


Para a análise dos dados recorreu-se ao programa Statistical Package for the Social Sciences (SPSS) versão 24.0. Foram calculadas frequências absolutas e relativas, medidas de tendência central (média) e de dispersão (desvio padrão). Foram ainda calculadas prevalências, expressas em percentagens com os respectivos intervalos de confiança a 95\% (IC95\%).

\section{RESULTADOS}

Quanto às variáveis profissionais, em média, o número de anos de trabalho em offshore foi de $10,07 \pm 5,52$ anos e na respetiva plataforma de África Subsaariana foi de $5,08 \pm 3,79$ anos. Pela análise da Tabela 2, a maioria dos trabalhadores referiu que trabalha em offshore à 2-4 anos; trabalha nesta plataforma à $1-4$ anos $(53,5 \%)$ e $81,8 \%$ dos trabalhadores pratica um sistema de rotação de 4-5 semanas.

Tabela2 - Variáveis profissionais dos trabalhadores da plataforma da África Subsaariana

\begin{tabular}{l|c|c}
\hline \multirow{2}{*}{$\mathbf{N}^{\mathbf{0}}$ anos em Offshore } & $\mathrm{n}$ & $\%$ \\
\cline { 2 - 3 }$\leq 1$ ano & 15 & 15,2 \\
$2-4$ anos & 43 & 43,4 \\
5-9 anos & 29 & 29,3 \\
$\geq 10$ anos & 12 & 12,1 \\
\hline Tempo na plataforma & & \\
\hline 1-4 anos & 53 & 53,5 \\
5-9 anos & 34 & 34,3 \\
$\geq 10$ anos & 12 & 12,1 \\
\hline Sistema de rotação & & \\
\hline 2 a 3 semanas & 18 & 18,2 \\
4 a 5 semanas & 81 & 81,8 \\
\hline
\end{tabular}

Pela análise da Tabela 3, a maioria dos trabalhadores mencionou que consome café (54,4\%); consome bebidas alcoólicas em terra $(83,8 \%)$, bebe moderadamente $(69,9 \%)$ e a bebida mais consumida é a cerveja (68,7\%); consome refrigerantes $(74,7 \%)$ sendo o mais consumido a coca-cola $(67,6 \%)$. Dos trabalhadores que consomem café, em média, bebem $3,37 \pm 1,83$ cafés por dia e dos trabalhadores que consomem tabaco, em média, fumam 15,64 $\pm 8,52$ cigarros por dia. Ao considerarmos o Índice de Massa Corporal (IMC) autodeclarado, a prevalência de pré-obesidade foi de $63,6 \%$ e de obesidade foi 19,2\%. Quanto à prática exercício físico, 45,5\% dos trabalhadores referiu praticar 'às vezes' exercício físico e 22,2\% 'frequentemente'.

Catorze trabalhadores disseram que 'sofrem de alguma doença', sendo a mais prevalente a hipertensão (42,9\%). Nos últimos 12 meses, 91,9\% dos trabalhadores consultou um médico em terra e 78,0\% referiu que a especialidade mais consultada foi de clínica geral; nos últimos 12 meses $72,0 \%$ dos trabalhadores assinalou consumir medicação para dormir e no último mês apenas $36,0 \%$ referiu tomar medicação para dormir. A maioria dos trabalhadores referiram que acordam cansados $(90,9 \%)$; sentem dificuldade em levantar-se de manha $(78,7 \%)$ e sentem dificuldade em manter-se acordados durante 0 dia $(59,6 \%)$. 


\section{ESTILOS DE VIDA EM TRABALHADORES OFFSHORE DE UMA PLATAFORMA DA ÁFRICA SUBSAARIANA}

Tabela 3 - Estilos de vida dos trabalhadores da plataforma da África Subsaariana

\begin{tabular}{|c|c|c|}
\hline \multirow[b]{2}{*}{ Consumo café } & $\mathbf{n}$ & $\%$ \\
\hline & & \\
\hline \multirow{2}{*}{$\begin{array}{l}\text { Sim } \\
\text { Não }\end{array}$} & 54 & 54,4 \\
\hline & 45 & 45,5 \\
\hline \multicolumn{3}{|c|}{ Consumo café após as 20 horas } \\
\hline Sim & 22 & 40,7 \\
\hline Não & 32 & 59,3 \\
\hline \multicolumn{3}{|c|}{ Consumo de álcool em terra } \\
\hline Sim & 83 & 83,8 \\
\hline Não & 16 & 16,2 \\
\hline \multicolumn{3}{|l|}{ Fumador } \\
\hline Sim & 22 & 22,2 \\
\hline Não & 77 & 77,8 \\
\hline \multicolumn{3}{|c|}{ Padrão de consumo de álcool em terra } \\
\hline Bebe pouco & 22 & 26,5 \\
\hline Bebe moderadamente & 58 & 69,9 \\
\hline Bebe em excesso & 3 & 3,6 \\
\hline \multicolumn{3}{|l|}{ Tipo de bebida } \\
\hline Cerveja & 57 & 68,7 \\
\hline Vinho & 17 & 20,5 \\
\hline Bebidas brancas & 9 & 9,1 \\
\hline \multicolumn{3}{|c|}{ Consumo de refrigerantes } \\
\hline Sim & 74 & 74,7 \\
\hline Não & 25 & 25,3 \\
\hline \multicolumn{3}{|l|}{ Índice de massa corporal } \\
\hline Peso normal & 17 & 17,2 \\
\hline Pré-obesidade & 63 & 63,6 \\
\hline Obesidade & 19 & 19,2 \\
\hline \multicolumn{3}{|l|}{ Pratica exercício físico } \\
\hline Sim, frequentemente & 22 & 22,2 \\
\hline Sim, às vezes & 45 & 45,5 \\
\hline Não & 32 & 32,3 \\
\hline
\end{tabular}

Tabela 4 - Variáveis clínicas dos trabalhadores da plataforma da África Subsaariana

\begin{tabular}{l|c|c}
\hline \multirow{2}{*}{ Sofre alguma doença } & $\mathbf{n}$ & $\mathbf{\%}$ \\
\cline { 2 - 3 } Sim & 14 & \\
Não & 85 & 14,1 \\
\hline Consultou médico (últimos 12 meses) & 91,9 \\
\hline Sim & 8 & 91,9 \\
Não & 18 & 8,1 \\
\hline Consumo medicamentos para dormir (últimos 12 m) \\
\hline Sim & 7 & 28,0 \\
Não & 90 \\
\hline Acordar cansado & 9 & \\
\hline Sim & 90,9 \\
Não & 78 & \\
\hline Dificuldade levantar manhã & 21 & 78,7 \\
\hline Sim & 21,3 \\
Não & 59 & \\
\hline Dificuldade em manter-se acordado durante dia & 59,6 \\
\hline Sim & 40 & 40,4 \\
Não
\end{tabular}




\section{DISCUSSÃO}

Pela evidência científica podemos constatar que o trabalho offshore tem particularidades em relação ao trabalho "em terra", é caracterizado por cargas horárias, sistemas de rotação e confinamento específicos. 0 sistema de rotação pode variar de empresa e localidade das plataformas. 0 trabalho offshore apresenta particularidades que remetem a um desempenho do trabalhador por dias consecutivos mensalmente ocorrendo o retorno a casa unicamente após o cumprimento do período de trabalho, o que desorganiza a vida psicossocial, familiar com implicações para a saúde (Antoniolli, Emmel, Ferreira, Paz \& Kaiser, 2015; Alvarez, Figueiredo \& Rotenberg, 2010). As doenças desenvolvidas pelos trabalhadores offshore resultam da exposição a riscos ocupacionais, confinamento, trabalho noturno, alterações do sono, estilos de vida, esforço físico (Antoniolli, Emmel, Ferreira, Paz \& Kaiser, 2015). Os estilos de vida que mais propiciam doenças nos trabalhadores embarcados derivam da alimentação inadequada, obesidade, fumo, álcool e sedentarismo (Antoniolli, Emmel, Ferreira, Paz \& Kaiser, 2015, Oenning, Carvalho \& Lima, 2014; Felipe-de-Melo, Silva, Assis \& Pinto, 2011). Destes hábitos sucedem as doenças, tais como, a doença cardiovasculares, síndrome metabólica, hipertensão arterial e musculosquelética (Felipe-de-Melo, Silva, Assis \& Pinto, 2011; Oenning, Carvalho \& Lima, 2014). No presente estudo a doença mais prevalente nos trabalhadores offshore foi a hipertensão.

Os resultados deste estudo, quanto aos estilos de vida corroboram a evidência científica. A maioria dos trabalhadores mencionou que consome café; consome bebidas alcoólicas, bebe moderadamente e a bebida mais consumida é a cerveja; consome refrigerantes, sendo o mais consumido a coca-cola; é fumador e em média, fumam 15,64 $\pm 8,52$ cigarros por dia; apresentam um $I M C \geq 25 \mathrm{Kg} / \mathrm{m}^{2}$ e $45,5 \%$ dos trabalhadores referiu praticar 'às vezes' exercício físico e 22,2\% 'frequentemente'.

Um estudo realizado no Brasil em empresas petrolíferas, com 210 trabalhadores (198 homens e 8 mulheres), com uma média de idade 32 anos, a maioria com 0 ensino médio (71,4\%), casados $(61,0 \%)$, com um tempo médio de trabalho no campo offshore de $5,9 \pm 5,6$ anos e com variação de 1 a 30 anos de experiência revelou, quanto às variáveis relacionadas com a saúde, que $11,0 \%$ dos trabalhadores considerou sua saúde regular/má; $29,5 \%$ eram fumadores; $79,5 \%$ possuía consumo de álcool de baixo risco; $11,9 \%$ consumo de risco; 1,4\% consumo nocivo; 0,5\% provável dependência ao álcool e a doença mais referida foi os problemas osteomusculares com uma prevalência de $15,0 \%$ (Vidal, 2014).

0 consumo de álcool e de tabaco poderá ser uma consequência de outros fatores psicológicos, alguns trabalhadores não conseguem controlar o stresse de trabalhar offshore a um ritmo exigente e por períodos prolongados, em condições de relativo confinamento e sujeitos a condições ambientais em constante mudança e entre os sinais de stresse dos trabalhadores, figura uma irritabilidade, ingestão excessiva de álcool ou tabagismo e consumo de drogas (Organização internacional do trabalho, 2017). Como refere o relatório para a segurança e saúde no trabalho na indústria do petróleo e do gás em países seleccionados da África Subsaariana de 2017, "0s trabalhadores que consomem drogas ou álcool, muitas vezes, fazem-no na ilusão de que essas substâncias ajudam a reduzir o stresse do trabalho, ou a conseguir boa-disposição, melhorar o desempenho, ultrapassar as pressões dos colegas ou conviver. No entanto, o abuso de substâncias, em geral, aumenta as probabilidades de acidentes e de absentismo, fazendo baixar a produtividade e o desempenho geral da empresa".

No que se refere às actividades de lazer, designadamente à prática de exercício físico, esta pode estar condicionada pelas próprias características do trabalho offshore, ao confinamento, ao espaço restrito e limitado, à própria alteração do ritmo do sono, à proximidade de atividades diferentes 


\section{ESTILOS DE VIDA EM TRABALHADORES OFFSHORE DE UMA PLATAFORMA DA ÁFRICA SUBSAARIANA}

como trabalho, alimentação, lazer, repouso. Souza (1996, p.2) refere que "0 trabalho em regime de confinamento dos trabalhadores offshore (plataformas marítimas, navios e submarinos) apresenta uma situação peculir que é a de estarem em alto mar, não raro, há centenas de quilómetros da costa, durante um período de vários dias, o que lhes permitem deslocarem apenas dentro de um espaço limitado. Vibrações, ruídos, conversas entre pessoas geralmente estão presentes em seus momentos de repouso, lazer ou refeições".

Relativamente às variáveis clínicas, os trabalhadores da presente investigação referiram que nos últimos 12 meses consultaram um médico em terra sendo a especialidade mais consultada "clínica geral'; consumiram medicação para dormir, têm dificuldade em levantar-se de manhã, acordam cansados e sentem dificuldade em manter-se acordados durante 0 dia. A evidência científica refere que os trabalhadores offshore apresentam privação do sono com sonolência (Alvarez, Figueiredo \& Rotenberg, 2010; Vidal, Abreu \& Portela, 2017; Antoniolli, Emmel, Ferreira, Paz \& Kaiser, 2015).

Estes trabalhadores estão expostos a vários riscos que os caracterizam de grupo profissional de risco para problemas de saúde e bem-estar.

\section{CONCLUSÕES}

Encontrámos na maioria dos trabalhadores desta plataforma da África Subsaariana uma elevada percentagem de estilos de vida não saudáveis. 0 trabalho em plataforma exige, tanto por parte do empregador quanto do trabalhador, atenção essencial à saúde e adoção de estilos de vida saudáveis no espaço offshore.

Do estudo realizado, podemos referir que é necessário implementar a promoção da saúde na procura por uma maior segurança e saúde dos trabalhadores.

\section{AGRADECIMENTOS}

Este trabalho é financiado por fundos nacionais através da FCT - Fundação para a Ciência e a Tecnologia, I.P., no âmbito do projeto UID/Multi//04016/2016. Agradecemos adicionalmente ao Instituto Politécnico de Viseu e ao CI\&DETS pelo apoio prestado.

\section{REFERÊNCIAS BIBLIOGRÁFICAS}

Alvarez, D., Figueiredo, M. \& Rotenberg, L. (2010). Aspectos do regime de embarque, turnos e gestão do trabalho em plataformas offshore da Bacia de Campos (RJ) e sua relação com a saúde e a segurança dos trabalhadores. Revista Brasileira de Saúde Ocupacional [Internet], 35(122), 201-216. Disponível em: http://www.scielo.br/pdf/rbso/v35n122/a04v35n122.pdf

Antoniolli, S.A.C., Emmel, S.V., Ferreira, G.E., Paz, P. O. \& Kaiser, D.E. (2015). Trabalho offshore e a atuação do enfermeiro embarcado: uma revisão integrativa. Revista da Escola de Enfermagem da USP, 49(4), 689-698. DOI: 10.1590/S0080-623420150000400021.

Castro, A. C., \& Nunes D. K. P. (2008). Análise Crítica do Gerenciamento de Stress em Plataformas Marítimas. In Anais do XXVIII Encontro Nacional de Engenharia de Produção. Rio de Janeiro.

Felipe-de-Melo, E.R.T., Silva, R.C.R., Assis, A.M.O. \& Pinto, E.J. (2011). Fatores associados à síndrome metabólica em trabalhadores administrativos de uma indústria de petróleo. Ciências \& Saúde Coletiva [Internet], 16(8), 3443-3452. Disponível em: http://www.scielo.br/scielo.php?script=sci_arttext\&pid=S1413-81232011000900012

Leite, R. M. D. S. C. (2009). Vida e trabalho na indústria de petróleo em alto mar na Bacia de Campos. Ciência \& Saúde Coletiva [Internet], 14(6), 2181-2189. Disponível em http://www.scielo.br/scielo.php?pid=S1413-81232009000600025\&script=sci_abstract\&tlng=pt

Maciel de Carvalho, M. (2010). "Vida e trabalho de marítimos embarcados do setor offshore". Tese de Mestrado em Saúde Pública. Escola Nacional de Saúde Pública. Rio de Janeiro. Disponível 
em http://pesquisa.bvsalud.org/sms/resource/pt/tes-4001

Oenning, N.S.X., Carvalho, F.M. \& Lima, V.M.C. (2014). Risk factors for absenteeism due to sick leave in the petroleum industry. Revista de Saúde Pública [Internet], 48(1), 103-122. Disponível em https://www.ncbi.nlm.nih.gov/pmc/articles/PMC4206122/

Organização internacional do trabalho. Departamento de Políticas Sectoriais. Segurança e saúde no trabalho na indústria do petróleo e do gás em países seleccionados da África Subsaariana. Relatório para discussão no Seminário tripartido da África subsaariana sobre segurança e saúde no trabalho na indústria de petróleo e gás (Maputo, Moçambique). Genebra 2017. ISBN 978-922-831324-6

Parkes, J. (2007). Reliability as argument. Educational Measurement: Issues and Practice, 26(4), 210.

Silva Júlio, D. I., \& Ferreira, M. C. (2007). Escala para avaliação de estressores ambientais no contexto off-shore-oil. Avaliação Psicológica, 6(2), 139-146. Disponível em: http://pepsic.bvsalud.org/scielo.php?script=sci_arttext\&pid=S1677-04712007000200004

Souza, A.A. (1996). Perfil do Homem offshore aspectos relevantes na relações no trabalho e familiares. Caderno de Pesquisa em administração, São Paulo, 1(3), 2º Sem.

Vidal, J.M. (2014). Saúde e padrão de consumo do álcool em trabalhadores Offshore. Dissertação de Mestrado. Universidade Federal do Rio De Janeiro, Escola de Enfermagem Anna Nery. Rio de Janeiro. Disponível em http://objdig.ufrj.br/51/teses/855299.pdf

Vidal, J.M., Abreu, A.M. \& Portela, L.F. (2017). Estresse psicossocial no trabalho e o padrão de consumo de álcool em trabalhadores offshore. Cadernos de Saúde Pública; 33(6):e00116616. Disponível em http://www.scielo.br/scielo.php?pid=S0102311X2017000606001\&script=sci_arttext

1 offshore - "afastado da costa" - está também relacionado com a atividade (prospeção, perfuração e exploração) de empresas de exploração petrolífera que operam ao largo da costa). 
